\title{
Arousing and managing astonishment; brief considerations on inter/cultural education in the classroom of French taught as a foreign language
}

\author{
[Susciter et gérer l'étonnement ; brèves considérations sur l'éducation \\ inter/culturelle en classe de français enseigné comme langue étrangère]
}

\author{
Jean-Claude Beacco
}

DOI: 10.18355/XL.2021.14.04.03

\begin{abstract}
Due to the polysemy of culture and inter-, intercultural education receives various interpretations in the teaching of French as a foreign language. Overall, they tend to subordinate "cultural" activities to language teaching, and they make little use of their educational potential. We propose here an interpretation of "interculturality" as education to otherness, which is central in the learning of a foreign language. It can take place on a regular basis in the classroom, in the form of specific teaching sequences and without any stated linguistic objectives. These "intercultural moments" in the language class have the role of arousing astonishment (if necessary) at societal otherness, as produced by "raw" (non-explanatory/descriptive) documents concerning singular facts. They should lead to group exchanges, which aim to soften egoethnocentric or alienating attitudes and to make these encounters with a difference an opportunity for personal development.
\end{abstract}

Key words: intercultural, education, foreign language, astonishment, otherness

\section{Résumé}

Du fait de la polysémie de culture et de inter-, l'éducation interculturelle reçoit des interprétations diverses en didactique du français langue étrangère. Globalement, elles tendent à subordonner les activités «culturelles » à l'enseignement de la langue et elles exploitent peu leurs potentialités éducatives. On propose ici une interprétation de «l'interculturel » comme éducation à l'altérité, l'altérité étant évidemment centrale dans l'apprentissage d'une langue étrangère. Elle peut se concrétiser de manière régulière en classe, sous la forme de séquences d'enseignement spécifiques et sans objectifs linguistiques affichés. Ces «moments interculturels » de la classe de langue ont pour rôle de susciter l'étonnement (si nécessaire) devant l'altérité sociétale, tel que produit par des documents «bruts » (non explicatifs/descriptifs) concernant des faits singuliers. Leur analyse doit conduire, dans le groupe, à des échanges qui visent à assouplir les attitudes égo-ethnocentriques ou d'adhésion aliénante et à faire de ces rencontres avec la différence une occasion de développement personnel.

Mots clés : interculturel, éducation, langue étrangère, étonnement, altérité

\section{Introduction}

La question de la didactique de l'« interculturel» à mettre en place dans les enseignements scolaires des langues étrangères (et, dans le cas présent, du français enseigné à des allophones) a déjà fait couler beaucoup d'encre et ce, depuis plusieurs décennies. J'ai d'ailleurs moi-même commis bien des articles et deux ouvrages sur le sujet (Beacco 2000, 2018). Ce bref exposé, qui reprendra des considérations présentées dans ces textes antérieurs, entend proposer, de manière simple et compacte, mon point de vue sur ce nœud de problèmes. On y décrira des pistes de réflexion destinées à fonder des démarches qui soient à la fois réalistes, utilisables de manière 
systématique en classe de français et qui cherchent à répondre à des finalités éducatives, comme celles désignées sous le terme d'éducation interculturelle.

\section{Les interprétations des rapports didactiques à établir entre langue et « culture» .}

La part inter/culturelle de l'enseignement/apprentissage des langues a fait l'objet de diverses interprétations didactiques fondées sur la polysémie intrinsèque de culture et de inter-. Mais, globalement, celles-ci cherchent à subordonner l'enseignement de la « culture » à celui de la langue et non à l'aborder de manière spécifique.

\section{Littérature}

Ainsi, depuis longtemps et aujourd'hui encore, « enseigner la culture » dans les cours de langue est interprété, dans la tradition humaniste, comme faire accéder les apprenants à textes littéraires (et, désormais, de plus en plus «paralittéraires ») dans la langue cible. Ce choix se fonde sur le fait que ces textes peuvent être considérés à la fois comme des réalisations exemplaires de la langue cible et comme des objets culturels significatifs... Apprendre auprès des «bons auteurs» permettrait ainsi de combiner langue et culture. Cependant l'entraînement à la lecture de ces textes ne peut être que limité : il n'est possible que sur des extraits et sans doute pas avant le niveau $\mathrm{B} 1$ pour les textes contemporains $\left(\mathrm{XIX}^{\circ}-\mathrm{XXI}^{\circ}\right.$ siècles) et à partir de $\mathrm{B} 2$ pour les textes de la période classique ou antérieurs à celle-ci. Ces textes ont toujours leur place dans l'enseignement, que ce soient de brefs poèmes (souvent d'auteurs contemporains) dès les niveaux $\mathrm{A} 1$ et $\mathrm{A} 2$ ou des extraits d'œuvres romanesques et davantage à des niveaux avancés comme B2. Mais l'utilisation pédagogique de textes reste circonscrite et elle n'est probablement pas en mesure de contribuer significativement aux acquisitions linguistiques nécessaires à la communication sociale ni même de faire «entrer», sauf exception, dans les œuvres du canon littéraire. L'entrée dans ces autres mondes de la fiction constitue bien une expérience interculturelle de l'altérité à gérer comme telle, mais on s'en tient souvent à ces contacts avec la culture cultivée.

\section{Lexique}

Une autre interprétation courante de la relation à établir entre langue et culture est de privilégier les entrées lexicales. Nous avons déjà discuté de cette option (formulée de manière compacte par le terme langue-culture) d'un point du point de vue de la didactique des langues (Beacco, 2000: 94-100) et cette problématique générale a aussi nourri une abondante réflexion (voir par ex. Scherzer 1987). Il est clair que des mots renvoient à des référents «culturels » soit parce que leur sens ne peut pas être établi en dehors des réalités sociétales auxquelles ils référent (les HLM ne sont pas seulement des logements loués peu cher; le pays de Caux ou celui de Bray ne sont pas des états), soit parce que les référents sont impliqués dans les pratiques sociales courantes (le muguet du premier Mai, la fève de la galette des Rois, les ponts entre les jours fériés...; voir récemment Le Français dans le Monde 2021 : Une approche culturelle des mots). Les mots servent alors de point de départ à l'apport d'informations de la part de l'enseignant.

On attribue aussi volontiers une valeur culturelle aux locutions de toute nature comme les réalisations attendues de certains actes de parole (à vos souhaits à côté de bless you (anglais), salute (italien), dii omen avertant (latin). C'est aussi le cas pour les combinaisons préférentielles: ces «expressions toutes faites», étudiées par I. Mel'čuk (en particulier, 1984 et suiv., 2013), sont des groupes nominaux comme : peinture fraîche (anglais wet painting), applaudissements nourris, mais aussi des locutions verbales comme prendre une décision (anglais make), bâtir des châteaux en Espagne, manger du lion, mener en bateau, ne pas avoir froid aux yeux, se faire sonner les cloches... Certaines comportent des traits linguistiques ou sociétaux anciens 
comme : c'est la croix et la bannière (renvoie aux processions), avoir maille à partir (maille : petite pièce de monnaie ; partir = diviser), mettre sa main au feu (renvoie aux ordalies médiévales), se mettre martel en tête (martel = marteau). C'est aussi le cas de la plupart des proverbes, eux aussi étant facilement considérés comme « culturels »... Ces dernières caractéristiques et surtout le fait que ces combinaisons de mots sont figées conduisent à les considérer comme de nature culturelle, puisqu'elles ne relèvent pas de la syntaxe (et donc de la compétence grammaticale des apprenants) mais d'une maîtrise idiomatique de la langue, propre aux locuteurs natifs et qui en définit l'identité langagière.

Cette interprétation lexicale de la part du «culturel » dans l'enseignement du français et des langues étrangères fait oublier que ce sont plutôt les usages mêmes de la langue qui sont à qualifier de «culturels": les manières d'aborder quelqu'un de le contredire, de prendre congé, de s'excuser... répondent à des habitudes et à des rituels différents d'une langue à l'autre, d'une communauté de communication à l'autre. L'étude de ces modalités, reconnues comme appropriées, de conduire les échanges verbaux oraux ou écrits, sont le propre de disciplines comme l'ethnolinguistique, l'anthropologie linguistique ou encore l'analyse contrastive des discours. Ces disciplines sont encore trop peu sollicitées en didactique des langues, car apprendre une nouvelle langue ne consiste pas seulement à s'approprier des sons et des formes, mais aussi à apprendre d'autres "manières" de se comporter dans l'usage des langues. Or, c'est là le principe fondateur dans l'approche communicative, qui ne se trouve vraiment pris en compte que dans quelques manuels récents se réclamant de l'éducation plurilingue. De ce point de vue, la culture n'est pas «dans » la langue »; ce sont ces comportements langagiers qui sont variables d'une communauté à l'autre et qui les caractérisent par des spécificités auxquelles la dénomination de culturelles semble adaptée.

\section{Connaissances « culturelles »/sociétales}

Une autre interprétation de la nature de la «culture » dans les apprentissages des langues étrangères relève elle aussi du lexique, mais dans une perspective strictement informative : le but est en fait de donner aux apprenants des informations sur la vie sociale ordinaire. Car celle-ci apparait sous de multiples formes dans les conversations courantes qui constituent souvent l'essentiel de l'apprentissage de l'oral : utiliser le small talk (= les échanges convenus, qui n'engagent à rien, portant sur la météo, la santé, la famille, les amis communs, le travail ...), acheter un objet ou un service, demander une information, demander son chemin, visiter un musée... Ces échanges quotidiens font ainsi référence à l'espace (feu-rouge, boulevard...) au temps social (les horaires des magasins, la rentrée de septembre, le 11 novembre...), à l'organisation de la vie collective (les PTT, le commissariat (de police), le permis (de conduire), la carte Vitale...), à l'actualité politique, économique, culturelle et sportive (Les Marcheurs, le PSG...). Ainsi en fonction des situations choisies (acheter dans une épicerie...) ou des scenarios sociaux (utiliser les transports en commun, consulter un médecin...), du vocabulaire doit être enseigné pour que l'on puisse être compris/comprendre et pour assurer la réussite de l'échange verbal. Cette forme (lexicale) pratique de la connaissance sociétale peut être elle aussi être qualifiée de culturelle, comme dimension de la sociologie de la vie quotidienne.

\section{Comparaison}

Cette attention lexicale portée à la connaissance des sociétés où se parle le français, dans ce qu'elles comportent d'utile à la communication, voire même à la vie au sein de celle-ci (comme touriste, comme stagiaire...) conduit à une certaine interprétation à la notion d' «inter-interculturel »: la comparaison. Celle-ci n'est d'ailleurs pas 
nouvelle, mais la vogue de «l'interculturel » apporte un surcroit de légitimité à cette démarche. En fait, «l'interculturel» est souvent réduit à des comparaisons sans préoccupation formative particulière. On est ainsi amené à mettre en regard des réalités sociétales (les fêtes, les vacances...) des comportements (la propreté), voire des attitudes ou des valeurs (racisme) pour en caractériser les ressemblances et les différences. Pour être fiables, ces activités devraient se fonder sur des sources d'information de qualité relatives aux pays où est utilisée la langue apprise. Il faut noter que, paradoxalement, celles-ci ne sont pas souvent convoquées pour le pays de résidence des apprenants et des enseignants, car on se fie volontiers pour cela à sa propre expérience ou à sa propre représentation des phénomènes concernés. Il convient aussi de s'assurer que les objets mis en relation sont bien comparables, même s'ils se ressemblent " en surface » : les économistes savent bien que le taux de chômage officiel n'est pas établi selon les mêmes critères d'un pays à l'autre et comparer le coût de certains biens identiques peut se révéler trompeur, car il est fonction de leur importance dans les usages sociaux : le vin en Grande-Bretagne est surtout consommé dans des circonstances festives, les légumes de consommation ordinaire en Europe le sont moins au Japon. De plus, dans certains cas, ces données sont malaisées à identifier: comment décider si les Français sont plus ou moins sympathiques que les Italiens ? On risque de s'en remettre à la notion de «mentalité nationale ", au statut épistémologique incertain en psychologie sociale. Des comparaisons bilatérales directes peuvent aussi fausser les perceptions, car elles devraient se faire dans des cadres généraux englobants (au moins un tertium comparationis) : la cuisine en France et la cuisine en Italie ou en Tunisie relèvent des catégories d'analyse communes utilisées pour décrire les pratiques alimentaires, faute de quoi on en restera à : bifteck frites vs pasta vs couscous. Et de telles mises en relation risquent de conduire à de généralisations (En France, on... Les Français sont... font ...) qui écrasent la diversité interne (géographique, sociale, économique culturelle...). Or la diversité interne d'un autre pays est fort comparable à la diversité interne de celui dans lequel on vit et dont on a conscience.

Quel est le but de ces comparaisons? «Faire parler» les apprenants (entreprise toujours délicate), leur donner des informations, les amener à réfléchir sur les limites de comparaisons de cette nature, exprimer une opinion, avec le risque que celle-ci relève du: c'est mieux là-bas/c'est mieux ici ou qu'elle se limite à enregistrer ressemblances ou différences ? Ou encore permettre de se situer soi-même ou réagir par rapport à des réalités autres ? Cette finalité est souvent invoquée, mais comme de manière rituelle, et l'on manque de données d'observations de classe pour en spécifier la nature. On perçoit bien que les comparaisons inter-sociétales, même superficielles, présentent un potentiel éducatif, mais celles-ci ne semblent pas souvent conçues dans la perspective de susciter et de gérer les réactions personnelles des apprenants avec l'étrangeté.

\section{Relation personnelle}

La finalité éducative d'impliquer les apprenants en tant que personnes dans l'apprentissage d'une langue est présente en didactique. On renvoie par là à la motivation intégrative (opposée à la motivation utilitaire, Gardner, Lambert, 1972), qui est celle de l'apprenant qui s'intéresse avec une certaine « ouverture d'esprit» à l'autre communauté linguistique/culturelle. L'accès à une autre langue sert des projets «extrovertis» qui peuvent conduire à l'assimilation (provisoire, réversible ou effective) à une autre communauté. Dans cet ordre d'idées, on a mis en évidence (Dreyer 2006) une motivation à l'apprentissage du français dite existentielle, comme attrait pour des manières d'être autres et qui sont rencontrées à travers cet apprentissage de la langue (mais qui ne sont pas nécessairement caractéristiques de la communauté autre). Cette forme d'investissement dans le cours de langue est susceptible de permettre aux apprenants de donner davantage de sens à leur existence 
sociale et individuelle: " la motivation existentielle affecte le rôle de l'autre (le groupe, l'étranger) qui n'agit plus alors sur l'apprenant comme une force centrifuge à travers diverses formes d'assimilation ou d'intégration. Elle tend au contraire à devenir une force centripète en incitant l'apprenant à se centrer sur son groupe d'appartenance, les apports de la langue-culture extérieure étudiée devenant une (res)source du développement personnel. Cette inversion des rôles, peut affleurer à la conscience des apprenants - certains étudiants choisissent délibérément d'intégrer les influences étrangères pour enrichir leur vécu - ou ils les laissent se produire à leur insu, même si nombre d'entre eux n'ont pas entrepris de réflexion sur leur relation avec l'étranger » (Dreyer 2006 : 38). On rencontre une préoccupation éducative du même ordre dans les réflexions didactiques et les pratiques de S. Ginet : il a fait réaliser à ses étudiants chinois de courts films autobiographiques à partir de documents disponibles dans leur famille et commentés en voix off et en français par eux-mêmes : «la frontière entre «le moi » et «l'autre », symbolisée par l'écart des langues-cultures, s'est estompé au profit d'un nouveau territoire, littéraire et cinématographique, un espace-temps de photos personnelles, intimement imprégnées de culture et de vie chinoises, raconté par une voix en français » (Ginet 2021). Cette recherche d'un mieux-être à soi par l'appropriation d'une langue extérieure constitue une des formes de truchement avec des sociétés autres qui conduit à répondre à des attentes personnelles des apprenants. Mais tous les apprenants ne sont pas disposés à s'impliquer dans de telles activités de nature aussi socio-affective. Et ces démarches « interculturelles» (crées par des enseignants singuliers) sont isolées et guère reproductibles mécaniquement. Elles témoignent clairement de la possibilité de donner aux activités « inter/culturelles » organisées en classe une valence éducative.

\section{2 Éduquer à l'altérité : pour une pédagogie de l'étonnement}

Les interprétations précédentes de «l'inter/culturel» sont parfaitement légitimes, tant que des activités de cette nature ne sont pas considérées comme constituant des ressources suffisantes pour des finalités éducatives qui ne se confondent pas avec des objectifs linguistiques.

L'éducation interculturelle est l'une de ces finalités. Elle constitue une dimension de l'éducation à la citoyenneté démocratique et elle est destinée à développer chez les apprenants la curiosité pour d'autres sociétés et d'autres manières de vivre. Elle vise à créer des attitudes ouvertes, pour que les apprenants puissent appréhender de manière positive et gérer de manière profitable les formes de contacts avec d'autres manières de vivre en société. Elle ambitionne d'assouplir les attitudes ego/ethnocentriques, pour amener à une gestion personnelle bienveillante de la diversité et pour faire considérer que ces contacts contribuent au développement de la personne. Cela implique que l'enseignant assume la responsabilité d'intervenir sur les attitudes, sur les croyances et les valeurs des apprenants pour les conduire vers des formes de prise en compte attentives de toutes les différences, du moins celles qui ne contreviennent pas visiblement à la Déclaration universelle des droits de l'homme.

Pour donner corps à cette éducation en classe de langue, nous sommes amené à considérer, après Coste et Cavalli $(2017,252)$, que l'éducation est un dispositif de médiation permettant aux apprenants d'accéder à des savoirs, des compétences et à des valeurs, en ce qu'elle a pour rôle de réduire les distances entre deux pôles en tension (par ex., entre les savoirs spontanés ordinaires et les connaissances savantes). De la sorte, l'École peut être conçue comme un espace de rencontre avec l'altérité (ce qui est autre, différent, étranger, inconnu...). Dans le cas de l'enseignement des langues étrangères, cette notion d'altérité (comme perception de ce qui est nouveau par rapport à soi-même et non dans l'absolu) est particulièrement centrale. Elle a vocation, de fait, à donner un sens éducatif à la notion d'interculturel : l'altérité de la

XLinguae, Volume 14 Issue 4, October 2021, ISSN 1337-8384, eISSN 2453-711X 
langue étrangère, des cultures/manières de vivre et d'organiser la vie collective de sociétés autres (au moins telle qu'elle transparait à travers les supports pédagogiques) est au centre de l'apprentissage langagier mais elle est aussi une " occasion de développement personnel » (Coste et Cavalli 2017, 251). La rencontre avec l'altérité sociétale/culturelle telle qu'elle s'effectue en classe de langue peut être pédagogiquement contrôlée (à la différence de ce qui se produit quand on regarde les informations télévisées, par exemple) et ainsi mise au service de l'éducation interculturelle à travers un « travail » sur les réactions qu'elle suscite. Tout enseignant de français a probablement eu l'occasion voir réagir ses élèves (par leur attitude ou verbalement) à certains traits de la société française, variables selon les contextes d'enseignement: par exemple, la laïcité de l'État, la colocation, l'interdiction de manger dans le métro parisien ou durant les cours, la structure de l'année (saisons, vacances scolaires, jours fériés...), le nucléaire, le Mont Saint Michel ... Et il a sans doute considéré cela comme des épisodes isolés ou sans particulière importance, alors que ces réactions de surprise, d'agacement, de rejet, d'adhésion ou d'admiration sont constantes (même si elles ne sont pas exprimées). Et elles peuvent devenir la matière même de son intervention éducative, en tant qu'elles manifestent les attitudes des apprenants devant l'altérité sociétale/culturelle.

On est ainsi amené à concevoir une "pédagogie de l'étonnement " qui permette de tirer un profit éducatif de cette forme d'émotion suscitée par ces rencontres avec de l'inconnu, de l'inhabituel, de l'inattendu ou de l'insolite. L'étonnement est d'ailleurs considéré comme le fondement de l'attitude philosophique, à partir des penseurs grecs et tout au long du développement ultérieur de la philosophie occidentale (Hersch 1993). C'est dire sa valence formative à l'interrogation et au questionnement de soi. L'étonnement peut d'ailleurs être suscité par l'expérience de formes internes et proximales de l'altérité, celles propres à l'espace social des apprenants. Ces différences perçues constituent des formes a priori acceptables de la diversité nationale, mais elles peuvent tout autant susciter des interrogations. A ce titre, elles sont à considérer comme des expériences interculturelles « internes ». Et il convient de mobiliser cette capacité, plus ou moins développée, des apprenants à s'interroger et à interpréter des faits sociaux inconnus dans leur propre espace social, culturel et linguistique pour la mettre au service de l'analyse d'expériences d'une altérité plus extérieure/plus «étrangère ». L'objectif n'est pas de créer de la surprise pour déclencher de l'intérêt ou un surcroît de motivation (par le dépaysement, l'exotisme ou le folklore), mais bien de rendre possible une certaine forme consciente de décentration.

Organiser délibérément des activités en classe de langue autour de l'étonnement a aussi l'avantage de rendre possible la création de séquences d'enseignement reproductibles, alors que bien des activités interculturelles proposées ne le sont qu'une seule fois, dans le cadre d'un dossier (par ex., à l'occasion du 11 novembre en France : la Guerre de 1914-1918 vue d'Allemagne et de France), d'un thème général (par ex., est-on plus libre en Angleterre qu'en France? Avez-vous la même conception de la liberté qu'en France ?) ou encore d'une autoanalyse (par ex., emploi de l'Autobiographie des rencontres interculturelle). Cela signifie que ces séquences sont spécifiques, à distinguer donc des autres formes de séquences didactiques (relatives à lecture, à la grammaire...). En particulier, elles ne répondent pas à des objectifs d'apprentissage de la langue, même si des bénéfices linguistiques indirects ne sont pas exclus.

Ces «moments interculturels» de la classe de langue se construisent selon une démarche reproductible :

rencontre avec la société autre (au moyen d'un document déclencheur à propos d'un fait de société) suscitant des réactions spontanées d'étonnement ou de curiosité de la part des apprenants, réactions qui 
trouvent sans doute souvent leur fondement dans des représentations stéréotypées ou égocentrées. Ces verbalisations sont à traiter immédiatement si elles sont très vives ;

- première gestion de cet étonnement: activités d'interprétation des apprenants à partir des données «brutes » pour reconstituer/comprendre le sens donné au fait de société retenu dans cette société même. Cette analyse menée par les apprenants est confrontée à des analyses et à des informations factuelles ayant une certaine valeur générale ;

gestion de l'étonnement (second temps) : après le détour informatif/réflexif, nouvelles verbalisations des apprenants exprimant leur point de vue/leur ressenti ; puis «travail» collectif sur ces réactions, par des échanges entre les apprenants ainsi qu'entre l'enseignant et les apprenants, pour faire passer ces derniers à des réactions plus contrôlées et plus réfléchies et, si possible, pour agir sur des attitudes d'adhésion, refus ou défiance systématiques aux différences. Ceci ne signifie pas que l'on entend les faire accepter ou approuver : ainsi, il ne s'agit pas de faire adhérer à la notion française de laïcité (qui conduit, par exemple, à interdire les signes religieux considérés ostentatoires dans l'enceinte des établissements scolaires), mais d'en faire comprendre, les origines, le fonctionnement, le rôle et la nature des débats auxquels elle donne lieu en France même.

Les rencontres avec des réalités distantes sont rendues possibles par des textes et des images, à la manière des reportages, des enquêtes, des films documentaires... que l'on « consomme » hors de l'École. Elles doivent concerner des faits singuliers ou bruts (événements ou caractéristiques de toute nature et non des analyses de ceux-ci). Elles sont conçues pour interpeller les apprenants, pour susciter leur curiosité et, par là, pour «remonter» à leurs croyances. Cette implication recherchée doit rester éducative, c'est-à-dire se garder à la fois de la provocation, de l'endoctrinement et du folklore. Les documents utilisés seront en langue cible.

Il faut se garder des différences qui ne font pas sens (les boîtes aux lettres sont jaunes en France et rouges au Royaume-Uni) et privilégier celles qui se rapportent à des faits sociétaux/culturels significatifs (par ex. le congé scolaire du mercredi/jeudi en France et la séparation de l'Église et de l'État). Les lieux de ces rencontres avec l'altérité sociétale peuvent être sélectionnés en fonction de l'âge des apprenants. Pour les plus jeunes, on peut sans doute retenir des faits de la vie quotidienne ordinaire et de ses aspects matériels (par ex., le classement du repas gastronomique des Français dans la liste du patrimoine culturel immatériel de l'UNESCO). Pour créer ces contacts, on peut aussi tirer parti d'événements qui se produisent dans le contexte des apprenants ; ceux-ci peuvent être des événements «critiques» intervenant dans le cadre des relations bilatérales entre les deux pays : par exemple, un épisode French bashing de la presse anglaise, rapporté dans la presse francophone, constitue un matériau possible pour la classe de français langue étrangère en Angleterre.

Gérer l'étonnement implique d'abord de faire accéder à des connaissances factuelles sur le phénomène considéré. Par là, on vise à développer des compétences d'interprétation de faits culturels et sociétaux plus élaborées que celles mises en jeu dans les analyses spontanées. Pour cela, on utilisera certains documents «bruts » : ils ne donnent pas d'informations générales mais sont des exemples ou des manifestations particulières ou partielles du fait considéré. Ces documents (en langue cible) pourront être identifiés, recherchés et examinés par les apprenants. Par exemple, pour faire "comprendre la cuisine française », on utilisera des menus de restaurants, les noms des différents types de boutiques alimentaires (charcuterie, fromagerie, boulangerie, rôtisserie, confiserie, épicerie fine), des sites de 
supermarchés, ... Ces séries de documents demanderont des analyses de la part des élèves, de manière à leur faire observer de près cette réalité et à tirer des informations raisonnablement généralisables de ces collections/corpus de données, peu significatives prises isolément. D'autres documents seront directement informatifs (comme des analyses de type économique de la consommation alimentaire des Français) et, bien entendu, adaptés au niveau de langue et à l'âge des apprenants (en fait, à leur expérience sociale). La compréhension de ces derniers documents (en langue cible ou en langue première) donnera lieu à un examen critique de la nature de ces sources d'information (article de presse, sondage d'opinion, statistiques...) et aussi à l'élaboration d'un cadre descriptif pour mettre en regard des réalités autrement que frontalement; et on passera, dans l'exemple choisi, de «comment mangent les Français ?» à «comment mangent les gens ? ». Il est alors indispensable d'avoir recours à des catégories descriptives générales comme chaud/froid, cru/cuit, végétal/animal, bouilli/rôti, doux/salé, individuel/collectif, simultané/successif, mets recherchés/tabous alimentaires... On construira ces catégories avec les élèves à partir de données précédentes et ils pourront les tester pour décrire eux-mêmes, par exemple " le repas du matin », dont le nom lui-même est variable d'une communauté à l'autre (voir des exemples de cette démarche dans Beacco \& Lieutaud, 1985 et sa théorisation dans Beacco, 2000). Cette recherche de documents est désormais possible grâce à l'Internet. L'accès aux informations et la prise de recul réflexif sont de nature à complexifier les réactions spontanées immédiates.

Pourquoi est-il ensuite fondamental de gérer l'étonnement au moyen des réactions verbales à l'altérité, suscitées par cette rencontre réaliste mais imaginaire (de fait, elle est sans enjeu effectif direct pour la personne) ? C'est parce que c'est cela qui permettra un " dialogue éducatif ». Le moment essentiel de la démarche proposée est celui des échanges verbaux (en langue première ou en langue cible au niveau B2 et plus) entre les apprenants et avec l'enseignant sur le fait de société découvert et analysé précédemment. Celui-ci prendra sans doute la forme d'interactions, qui seront plus ou moins consistantes, en fonction de la pertinence du sujet retenu pour les apprenants et de leur capacité à interroger leurs représentations. Il ne s'agit aucunement de «faire parler les élèves en langue étrangère », mais de leur donner la parole en tant que personnes. Cet échange (qu'il est inutile de faire durer artificiellement du point de vue qui nous occupe) pourra aussi être organisé par la recherche d'argumentations adéquates relatives aux différents points de vue exprimés sur le sujet abordé. Il vise l'établissement d'un consensus acceptable ou la prise de conscience par chacun de ses propres postures. De ce point de vue, toutes les opinions ne sont pas acceptables et c'est là que commence le travail sur les représentations sociales exclusivistes et les attitudes de rejet non discriminé de l'altérité. L'enseignant est modérateur et médiateur mais il est surtout dépositaire des valeurs sociales fondatrices que l'École a la responsabilité de transmettre ; tout enseignant de langue a la responsabilité, comme l'ensemble des enseignants, de faire comprendre et accepter les valeurs fondatrices du vivre-ensemble démocratique.

M. Gout (2015) souligne l'intérêt de tels échanges pour la formation linguistique des personnes migrantes : "L'exercice consiste à exprimer ses sentiments sur [un] sujet [faisant partie du vécu] et à essayer de comprendre les comportements. Des débats ont lieu dans la classe et le but est de mettre à distance ses représentations, ses préjugés, et de s'ouvrir à la nouveauté, à la diversité. » (2015 : 311). Les opinions des apprenants se fondent sur des arguments d'origines diverses, qui relèvent de l'opinion, des représentations sociales circulantes ou de l'expérience personnelle. Quand ils portent sur des faits de société pour lesquels des données sont disponibles, les échanges possibles peuvent prendre en compte celles-ci et elles tourneront autour des interprétations à en donner. Mais, le plus couramment, les échanges sur des questions de société relèvent davantage de points de vue, d'idées reçues et de convictions où les positions établies valent souvent davantage que les faits avérés. Il importera alors 
d'essayer d'établir la consistance des faits au moyen de données observables, quand elles sont disponibles. Savoir si «les Anglais » ou « les Japonais » sont plus nationalistes que " les Français » suppose de s'accorder sur la nature des manifestations de cette idéologie et d'en estimer le «poids relatif ». L'affaire n'est pas simple et elle doit sans doute conduire le groupe à reformuler la question autrement : qui accuse qui d'être nationaliste et dans quelles circonstances ?

\section{Conclusion}

Nous avons cherché à décrire très brièvement des activités éducatives fondées sur la prise en charge de l'étonnement devant les manières de "faire société » d'autres communautés humaines (activités qui sont l'une des formes possibles à donner à l'éducation interculturelle). Ces activités ne sont pas étrangères à l'expérience des enseignants, car des échanges entre apprenants à propos de faits de société non familiers peuvent surgir, de manière imprévisible, à propos de n'importe quel document utilisé pour enseigner la langue. Même si les enseignants n'ont pas identifié cet « événement de classe » particulier (qui n'est pas accidentel), ils ont peut-être développé une certaine expertise professionnelle qui leur permet d'y faire face et de les gérer dans le sens indiqué ici. Mais nous ne disposons pas d'assez de données et d'analyses dans ce domaine. Cette démarche devra être précisée et, d'une certaine manière, validée par des observations de terrain et des entretiens avec les enseignants et les apprenants qui y auront participé. Mais il ne faut pas négliger le fait que les facteurs contextuels sont nombreux qui conduisent responsables éducatifs, enseignants et certains apprenants (adultes) eux-mêmes à considérer cette démarche comme peu acceptable: "on n'est pas là pour faire de la politique », dira-t-on facilement.

Avant tout, il importe que les enseignants retrouvent le sens profond de la notion d'interculturel, terme trop banalisé ; qu'ils soient davantage conscients de la nature de l'étonnement de leurs apprenants devant l'altérité ; qu'ils soient préparés à accueillir leurs réactions; et qu'ils soient outillés pour en faire un moment d'apprentissage spécifique, car ce ne sont pas des activités d'enseignement de même nature que les autres (elles ont une cohérence pédagogique propre). Autrement dit, ceci doit permettre qu'ils y voient une occasion de former les apprenants à une perception plus apaisée et bienveillante des autres. La contribution de chacun sera sans doute modeste, en considérant le volume horaire d'enseignement disponible à consacrer à de telles séquences inter/culturelles. Mais il est hors de doute que chacun dispose là d'un moyen supplémentaire de contribuer, à sa manière, à rendre les apprenants plus conscients de ce qui permet aux hommes et aux femmes, tous égaux et tous différents, de vivre ensemble dans la cité.

\section{Bibliographic references}

BEACCO, J.-C. 2000. Les dimensions culturelles des enseignements de langue. Des mots aux discours, Paris : Hachette.

BEACCO, J.-C. 2018. L'altérité en classe de langue. Pour une méthodologie éducative, Paris : Didier.

BEACCO, J.-C. 2019. L'éducation culturelle interculturelle dans l'enseignement du français à l'École. In : Conférence prononcée au Séminaire international de l'Association Nationale de Enseignants de Français du Liban (Beyrouth et Tripoli, 1112 mai 2018. Available online:

https://www.academia.edu/40410134/L\%C3\%A9ducation_culturelle_interculturelle_ dans_lenseignement_du_fran\%C3\%A7ais_\%C3\%A0_1\%C3\%A9cole

BEACCO, J.-C. - LIEUTAUD, S. 1985. Tours de France. Travaux pratiques de civilisation, Paris : Hachette, manuel (304 p.) et guide du professeur (174 p.). 
CONSEIL de l'Europe. 2009. Autobiographie des rencontres interculturelles, Strasbourg : Conseil de l'Europe. Available online :

https://www.coe.int/fr/web/autobiography-intercultural-encounters

COSTE, D. - CAVALLI, M. 2017. Altérité, communauté, médiation, mobilité : des notions à manipuler avec précaution. In : Jeoffrion, C. \& Narcy-Combes, M.-F : Perspectives plurilingues en éducation et formation. Des représentations aux dispositifs, Rennes : Presses universitaires de Rennes, pp. 243-256.

DREYER, S. 2006. Affectivité et identité dans les cours de langue. Le cas de l'enseignement du français dans les universités de Taiwan. Thèse pour le doctorat, Université du Maine.

GARDNER, R. - LAMBERT, W. 1972. Attitudes and motivation in second language learning, Rowley : Newberry House.

GINET, S. 2021. Écriture de soi : émergence du singulier culturel dans la langue de l'autre. In: Actes du Colloque international: Carrefours culturels, Université des langues de Pékin (BFSU), in press.

GOUT, M. 2015. Le rapport entre langue et intégration dans les dispositifs linguistiques pour migrants nouveaux arrivants en Allemagne, Belgique, France et Royaume-Uni, thèse pour le doctorat, Université d'Aix-Marseille.

HERSCH, J. 1981, transl. In French 1993. L'étonnement philosophique. Une histoire de la philosophie, Paris : Folio Essais/Gallimard.

LE FRANÇAIS dans le Monde. 2021. Cher lexique. Une approche culturelle des mots, $n^{\circ} 435$-juillet.

MELCUK, I et al. 1984, 1988, 1992, 1999. Dictionnaire explicatif et combinatoire du français contemporain (I à IV), Montréal : Les Presses de l'Université de Montréal.

MELCUK, I. 2013. Tout ce que nous voulions savoir sur les phrasèmes, mais... In: Cahiers de lexicologie vol. 102, n. 1, pp. 129-149.

SHERZER, J. 1987. A Discourse Centered Approach to Language and Culture. In: American Anthropologist, vol. 89, n. 2, pp. 295-309 ; transl. In French 2012. In : Langage et société, vol. 139, pp. 21-45.

Words : 5771

Characters : 38071 (21,15 standard pages)

Prof. Dr. Jean-Claude Beacco

Université Sorbonne nouvelle - Paris 3

La Chancellerie des Universités de Paris

47, rue des Écoles

75230 Paris Cedex 05

France

jcb.mdg@wanadoo.fr 